\title{
Editorial
}

\section{Prenatal Diagnosis}

Prenatal diagnosis are the spectrum of prenatal care for detecting anatomic and physiologic problems with the zygote, embryo or fetus as early as possible. It can be done before starting of gestation, as in preimplantation genetic diagnosis (PGD) or as early in gestation as possible.

The problems which are detectable by the use of prenatal tests are neural tube defect, chromosomal abnormalities like down syndrome and gene mutation which lead to genetic disorders and birth defects like spina bifida, cleft palate, Tay-Sachs disease, sickle cell anaemia, thalassemia, cystic fibrosis, muscular dystrophy and fragile $X$ syndrome.

\section{What is the purpose of prenatal diagnosis?}

There are three purposes of prenatal diagnosis: (1) to enable timely medical or surgical treatment of a condition before or after birth, (2) to give the parents the chance to abort a fetus with the abnormality and (3) to give parents the chance to prepare themselves psychologically for a baby with a health problem or disability, or for the likelihood of a stillbirth.

Indications of prenatal diagnosis:

1. Women over the age of 35

2. Women who have previously had premature babies or babies with a birth defect, especially heart or genetic problems

3. Women who have family histories or ethnic backgrounds prone to genetic disorders, or whose partners have these

4. Women who are pregnant with multiples (twins or more)

5. Women who have history of recurrent miscarriages.

It can be done after establishing or before establishing the pregnancy.

After establishing the pregnancy the tests are divided in two aspects.

1. Prenatal screening (Non-invasive tests)

2. Prenatal diagnostic tests (Invasive tests)

Prenatal screening tests (Non-Invasive tests):

Prenatal screening offers two types of screening tests to pregnant women in order to identify individuals who are at increased risk for carrying a fetus with a specific birth defect.

a) Ultrasonography

b) Biochemical marker tests

a) Ultrasonography: Ultrasonography at early weeks to detect nuchal translucency in the fetus, which is associated with increased risk of chromosomal abnormality and other diseases ${ }^{1}$. Sonographic measurement of the thickness of the nuchal fold between 11 and 13 weeks of pregnancy, together with maternal age and biochemical markers allows an individualized risk of aneuploidies such as trisomy 21,13 , and 18 to be calculated. Operator's qualification, choice of appropriate duration of investigation, technical considerations and inclusion of additional parameters from $2^{\text {nd }}$ trimester (around 20-22 weeks) 4D ultrasonogram such as measurement of the nasal bone, fingers, Doppler assessment of the tricuspid valve and the ductus venosus, and the facial angle allows individualized detection rates for trisomy 21 to be increased to up to $95 \% 2,3$.

b) Biochemical markers:

1. Triple marker tests: It is usually done between 15 and 20 weeks by measuring

i) Alpha fetoprotein (AFP),

ii) HCG and

iii) Free estriol (E3)

2. Quadruple test when Inhibin A is added with triple test is called quadruple test.

3. In recent years, the measurement of human chorionic gonadotrophin (HCG) and pregnancyassociated plasma protein A (PAPP-A) in maternal serum between the 11th and 14th weeks of pregnancy have become increasingly established in combination with nuchal translucency measurement and maternal age (combined first trimester test) ${ }^{4}$.

4. Cell free DNA testing: Based on DNA of fetal origin circulating in the maternal blood can be tested to identify aneuploidy ${ }^{5}$. Beginning in 2011 
this test is available in USA by which gender of a fetus as early as at six weeks can be diagnosed. Cell-free fetal DNA also allows whole genome of the fetus, thus determining the complete DNA sequence of every gene ${ }^{6}$.

\section{Prenatal diagnostic tests (Invasive tests)}

Indications of invasive tests:

- Maternal age: Probability of chromosomal anomalies increases with maternal age. Around half of chromosomal anomalies are accounted for by trisomy 21 (Down syndrome)

- Following an abnormal result from non-invasive screening

- Following an ultrasound finding which raises the possibility of a chromosomal problem

- In the presence of a known translocation, inversion or insertion in one parent; in these cases, the probability of an unbalanced aberration is increased, over and above the maternal age-related risk.

- A chromosomal anomaly in an existing child of the couple's. For example, after the birth of a child with free trisomy, the risk of a numerical chromosomal abnormality is increased by $1 \%$ for each subsequent child compared to other parents of the same age.

Following tests are done according to gestational age

1. Chorionic villus sampling between 11-14 weeks.

2. Amniocentesis between 15-17 weeks.

3. Placental biopsy from 15 weeks.

4. Cordocentesis from 16-20 weeks.

5. Fetal biopsy from 20 weeks for diagnosis of specific genetic dermatoses.

\section{Before establishing pregnancy:}

Preimplantation genetic diagnosis (PGD)

Preimplantation diagnosis (PID) is carried out on embryonic cells or from gamete prior to the establishment of pregnancy. This requires collection of eggs and in vitro fertilization (IVF) or intracytoplasmic sperm injection (ICSI).

\section{Tests are}

1. Polar body biopsy : By taking polar body from oocyte or from fertilized egg where mother has some defect. Drawback is it can give information only from maternal genetic transformation.

2. Embryonic cell biopsy: One cell (blastomere) is removed from 8 cell embryo or trophoectodermal cell is removed from blastocyst for molecular cytogenetic or molecular genetic analysis.

The applications of PGD are:

- The detection or exclusion of a specific unbalanced chromosome translocation, where one parent carries a Robertsonian or reciprocal translocation.

- The detection or exclusion of a particular mutation in the presence of an increased risk of a monogenetically inherited disease.

\section{References:}

1. Wald NJ, Morris JK, Walker K, Simpson JM. Prenatal screening for serious congenital heart defects using translucency: a meta-analysis. Pren Diagn. 2008;28:1094-1104.

2. Nicolaides K. Some thoughts on the true value of ultrasound. Ultrasound Obstet Gynecol. 2007;30:671-674.

3. Cicero S1, Bindra R, Rembouskos G, Spencer $\mathrm{K}$, Nicolaides $\mathrm{KH}$. Integrated ultrasound and biochemical screening for trisomy 21 using fetal nuchal translucency, absent fetal nasal bone, free beta-hCG and PAPP-A at 11 to 14 weeks. Prenat Diagn. 2003;23(4):306-10.

3. Lao, M.R.; Calhoun, B. C.; Bracero, L. A.; Wang, Y.; Seybold, D. J.; Broce, M.; Hatjis, C. G. The ability of the quadruple test to predict adverse perinatal outcomes in a high-risk obstetric population. J Med Screen. 2009;16 (2): 55-59.

5. Fan HC, Blumenfeld YJ, Chitkara U, Hudgins L, Quake SR; Blumenfeld; Chitkara; Hudgins; Quake. Non invasive diagnosis of fetal aneuploidy by shotgum sequencing DNA from maternal blood. Proc. Natl. Acad. Sci. U.S.A. 2008; 105 (42): 16266-71.

6. Yurkiewicz, I. R.; Korf, B. R.; Lehmann, L. S. Prenatal whole-genome sequencing -is the quest to know a fetus's future ethical?. New England Journal of Medicine. 2014; 370 (3): 195-7

\section{Dr. Rashida Begum \\ Editor}

